\title{
Recent Trends of Sero-Prevalence of Syphilis in Patients Attending a Tertiary Care Centre in North India
}

\author{
Parul Punia*, Deepinder Singh, Kiran Bala, Aparna, Uma Chaudhary and Akshit Griwan \\ PGIMS, Rohtak, Haryana, India \\ *Corresponding author
}

\begin{abstract}
Keywords
Precooling, Storage, Temperature and Shelf life.

Article Info

Accepted:

10 September 2017

Available Online:

10 November 2017 Maternal syphilis is associated with stillbirths and spontaneous abortions in number of cases. Diagnosis of syphilis is dependent on antibody detection by non treponemal/ cardiolipin (screening test) and treponemal tests (confirmatory tests). Material and Methods: A total of 28,407 serum samples obtained from patients of various departments from January 2011 to December 2015. All the serum samples were screened by VDRL testing and confirmation was done by TPHA test. Results: Seroprevalence of syphilis in this study was $0.68 \%$. Year-wise prevalence was $0.87 \%$ in $2011,0.71 \%$ in $2012,0.75 \%$ in $2013,0.45 \%$ in 2014 and $0.67 \%$ in 2015 . Seroprevalence of patients attending the skin and veneral disease clinic, obstetrics and gynaecology clinic and other departments were $3.27 \%, 0.35 \%$ and $2.23 \%$ respectively. Maximum prevalence was found in the sexually active age group i.e., 21-33 years. Conclusion: Various studies in India have shown similar rates of seroprevalence in the non high risk groups. A similar study in our institute done in the previous 5 consecutive years had shown higher rates of seroprevalence. The decreasing trend could be attributable to improved programs for prevention and management of STDs as well as availability of treatment in STD clinics. It is recommended to screen ANC cases for svphilis antibodies for earlv detection and prevention of complications.
\end{abstract}

A B S T R A C T

Syphilis is a sexually transmitted disease (STD), caused by Treponema pallidum. It not only has significant implications on public health but also facilitates the acquisition of other STDs such as HIV infection as well as complicates pregnancy related outcomes.

\section{Introduction}

Syphilis is a sexually transmitted disease (STD) that is caused by spirochaete Treponema pallidum. The route of transmission of the disease is mainly through sexual intercourse including the genital, oral, and anal routes but it can also be transmitted via blood transfusion and via contaminated needles in intravenous drug users. ${ }^{1}$ This disease not only has significant implications on public health but also complicates pregnancy related outcomes. ${ }^{2}$
Maternal syphilis is amongst the leading causes of perinatal morbidity and mortality and is documented to be associated with stillbirths and spontaneous abortions in large number of cases. In the developing countries, an estimated 460000 abortions, stillbirths and 27000 cases of congenital syphilis have been documented globally. ${ }^{3,4}$ The survivors are further at risk of various long-term sequelae such as cataract, meningo-encephalitis, learning disabilities etc. ${ }^{5}$ Moreover, it has also 
been found to facilitate the acquisition of other STDs including HIV infection (World Health Organization, 2001). ${ }^{6}$

World over, excluding HIV, there are 333 million new cases of STIs per year. Sexually transmitted infections are far more common in developing countries than industrial countries. ${ }^{7}$ The sero-prevalence rates are extremely varied amongst different countries and different subpopulations of the same country as well. In a report, based on data from for 2005 to 2012, the global prevalence for syphilis was estimated to be $0.5 \%$ with regional values ranging from $0.2 \%$ to $1.8 \%{ }^{8}$ Various studies done in India have also shown varied rates of syphilis ranging from $5.4 \%$ to $8.2 \%$ amongst STD clinics and $0.84 \%$ to $0.98 \%$ in antenatal groups. ${ }^{9,10}$ In one such study done in central India, prevalence of syphilis, as high as $21.9 \%$ was seen in long distance truck drivers. ${ }^{11}$

The diagnostic tests for syphilis are relatively simple to perform. These include antibody detection by various non treponemal/ cardiolipin (used for screening of syphilis patients) and treponemal tests (confirmatory tests). ${ }^{12}$ The Center for Disease Control recommends the use of a combination of a non-treponemal test and a treponemal test for screening and diagnostic purposes. ${ }^{13}$

Syphilis is a sexually transmitted disease, if diagnosed and treated timely can be successfully treated and controlled by effective and stringent public health measures. Hence, this study was designed to estimate and understand the prevalence of syphilis in our region as there is paucity of studies reported regarding sero-prevalence in different sub-populations of syphilis in India. Present study would help in framing and implementing effective STD control strategies.

\section{Materials and Methods}

It was a retrospective study conducted in the Department of Microbiology, Pt. B.D. Sharma Post Graduate Institute of Medical Sciences, (PGIMS), Rohtak Haryana (India). A total of 28,407 serum samples obtained from patients attending outdoors and indoors including Obstetrics and Gynaecology, Skin and VD, Medicine, Surgery and other clinical departments of PGIMS from January 2011 to December 2015 were included in the study. Any sample received from the ART clinic was excluded. All the serum samples were first subjected to qualitative VDRL test and further quantitative VDRL test using various serum dilution was done for reactive samples. The VDRL antigen was obtained from Laboratories of Serologist, Kolkata, India. The samples having titer of more than 1:8 were further subjected to Treponema pallidum Haemagglutination assay (TPHA), which is a treponemal specific test, to confirm the results.

\section{Antigen preparation ${ }^{14}$}

The VDRL antigen, which was provided with the kit, was prepared according to the manufacturers' instructions. $0.4 \mathrm{ml}$ of buffered saline was pipetted out in $30 \mathrm{ml}$ round bottle. Antigen $(0.5 \mathrm{ml})$ was added drop by drop to the buffered saline while continuously rotating the bottle on a flat surface over a period of 6 seconds. After the last drop was blown out, the bottle was shaken for another 10 seconds (Winn et al., 2005).

\section{Qualitative VDRL test $^{14}$}

The glass slides $(2 \times 3$ inches $)$ with 12 paraffin rings of approximately $14-\mathrm{mm}$ inside diameter were taken. Serum $(0.05 \mathrm{ml})$ was added into one ring and a drop (1 of $60 \mathrm{ml}$ ) of antigen was added to the serum. Serum and 
antigen were mixed with a wooden stick, and the slide was rotated for 4 minutes on a mechanical rotator set at 180 rounds per minute.

The tests were read immediately after rotation under a microscope with the low power objective (100× magnification). The results were read as non-reactive when there were no clumps or every slight roughness, weekly reactive when small clumps were observed and reactive when medium to large clumps were observed (Winn et al., 2005).

\section{Quantitative VDRL test $^{14}$}

A quantitative test was performed on all reactive serum samples. Successive two fold dilutions of the serum were made in 0.9 percent saline. Each dilution was treated as an individual serum and tested as described under a qualitative VDRL test. The results were reported in terms of highest dilutions which gave a frank reactive reaction (Winn $e t$ al., 2008)

\section{Results and Discussion}

Out of 28,407 serum samples received in the five year study period from January 2011December 2015, $196(0.68 \%)$ were found to be sero-positive for syphilis. Year-wise prevalence was $0.87 \%$ in $2011,0.71 \%$ in $2012,0.75 \%$ in $2013,0.45 \%$ in 2014 and $0.67 \%$ in 2015. The year-wise seroprevalence observed in various departments is shown in Table 1. Highest sero-prevalence was seen in patients attending the skin and veneral disease $(3.27 \%)$ clinic followed by surgery and medicine departments (2.23\%) followed by obstetrics and gynecology clinic $(0.35 \%)$. Maximum prevalence was found in the sexually active age group i.e, 21-30 years $(0.94 \%)$. The age wise prevalence is shown in Table 2.

A similar consecutive five year (2005-2010) study was conducted in our institute and the sero-prevalence was observed to be $1.83 \%$. On statistical analysis, this was found to be significantly higher than the sero-prevalence observed in the present study ( $\mathrm{p}$-value < 0.00001 )

In the present study, the five year seroprevalence rate was found to be $0.68 \%$. Various studies in India have shown variable rates of sero-prevalence due to the different subgroups included in their studies, ranging from $21 \%$ in the truck drivers to $1.9 \%$ in ANC group. ${ }^{15}$

\begin{tabular}{|c|c|c|c|c|c|c|}
\hline \multicolumn{7}{|c|}{ Table.1 Trends of VDRL Reactivity from 2011 to 2015 in PGIMS, Rohtak } \\
\hline \multirow[t]{2}{*}{ Departments } & \multicolumn{5}{|c|}{ Number of VDRL Reactive Cases } & \multirow{2}{*}{$\begin{array}{l}\text { Reactive } \\
\text { Samples } \\
(\%)\end{array}$} \\
\hline & 2011 & 2012 & 2013 & 2014 & 2015 & \\
\hline $\begin{array}{l}\text { Obstetrics \& } \\
\text { Gynaecology }\end{array}$ & 17 & 21 & 23 & 7 & 20 & $0.35 \%$ \\
\hline Skin \& VD & 31 & 14 & 18 & 19 & 20 & $3.27 \%$ \\
\hline $\begin{array}{l}\text { Medicine, } \\
\text { Surgery }\end{array}$ & 0 & 3 & 2 & 0 & 5 & $2.23 \%$ \\
\hline $\begin{array}{l}\text { Total } \\
\text { Samples } \\
\text { received }\end{array}$ & 5469 & 5368 & 5721 & 5763 & 6086 & 28,407 \\
\hline $\begin{array}{l}\text { Reactive } \\
\text { Samples }\end{array}$ & $48(0.87 \%)$ & $38(0.71 \%)$ & $43(0.75 \%)$ & $26(0.45 \%)$ & $41(0.67 \%)$ & $\begin{array}{l}196 \\
(0.68 \%)\end{array}$ \\
\hline
\end{tabular}




\begin{tabular}{|l|l|l|}
\hline \multicolumn{3}{|c|}{ Table.2 Seroprevalence of syphilis among different age groups } \\
\hline Age Groups & Total no. of samples & Reactive samples \\
\hline$<19$ yrs & 938 & $8(0.85 \%)$ \\
\hline $20-30$ yrs & 18013 & $171(0.94 \%)$ \\
\hline $30-40$ yrs & 4123 & $9(0.22 \%)$ \\
\hline $35-40$ yrs & 4234 & $6(0.14 \%)$ \\
\hline$>40$ yrs & 1062 & $2(0.18 \%)$ \\
\hline
\end{tabular}

Amongst the three groups that were included in the study, the highest prevalence $(3.27 \%)$ was noted in the patients attending skin and venereal disease clinic. This could be attributed to the fact that it represents a specified sub-population who came with suspected symptoms of venereal disease in comparison to the ANC group which represents the normal healthy population without any suspicion of any STD.

But this prevalence rate is lower than various other studies done in our country who have documented varying rate ranging from 5.4\%$8.2 \% .^{10,11}$ This highlights the success of programs being implemented for prevention and management of STDs at STD clinics in our institute.

The prevalence in the ANC group, which represents the normal healthy population, in our study was $0.35 \%$ which is in concordance to another study done by Hira Kumar et al., who acknowledged $0.28 \%$ ANC population to be seropositive for syphilis. ${ }^{5}$ Various other studies from India on antenatal group have shown slightly higher prevalence varying from- $0.84 \%$ $0.98 \% .^{9}$ Though the prevalence is lower in our region, the diagnosis of syphilis is still valuable in pregnancy because if left untreated, it may lead to a number of complications like premature delivery, repeated abortions, still birth as well as development of congenital syphilis in the neonates.

Patients from OPDs or admitted to other wards with features suggestive of secondary or tertiary syphilis were screened and sero-prevalence of this group was $2.23 \%$ which is similar with the study by Khan et al., ${ }^{16}$
The highest seroprevalence was seen in the age group 20-30 years which is the sexually active age group, and hence is at risk of acquiring sexually transmitted diseases. Similar trend has been noted by Maity et al., who also documented highest prevalence in the sexually active age group. ${ }^{10}$ Hence, this population must also be screened as they may be responsible for further transmission of this disease by sexual means as well as by blood donation, since they also represent the healthy blood donor group.

A similar consecutive five year study (20052010) was conducted in our department and the sero-prevalence observed was $1.83 \%$ which is significantly higher than the rates observed in the present study i.e., $0.68 \% .^{17}$ this statistically significant declining trend observed in the consecutive five years again points towards the success of improved STD controls programmes being implemented in our hospital.

Syphilis is a classic example of STD that can possibly be controlled easily as well as treated effectively and economically, by implementing efficacious public health measures. There are number of feasible and cost-effective diagnostic tests available for the diagnosis of this disease. Hence, the rates of prevalence of syphilis in different geographic areas must be utilized for formulating strategies to control it, so that the complications associated with it may be prevented.

\section{References}

1. Olokoba, A.B., Olokoba, L.B., Salawu, F.K., et al., (2009): Syphilis in voluntary blood donors in north-eastern Nigeria. Eur. J. Sci. Res., 31, 335-340. 
2. Barde P S, Tankhiwale N S. Seroprevalence of syphilis in ante natal cases in Central India. International journal of medical science and clinical inventions 4(8): 31433145, 2017

3. Nwokedi EE, Iliyasu Z, Dikko AU, Azeez AO, Mohammed B. Syphilis in a Nigerian paramilitary agency: need for treatment policy. Ann Afr Med 2005; 4(4): 177-179.

4. Lumbiganon P, Piaggio G, Viller J, Pinol A, Bakketeig L, Bergsjo $\mathrm{P}$, et al., The epidemiology of syphilis in pregnancy. Int $\mathbf{J}$ STD AIDS 2002; 13: 486-494

5. Patel K H, Patel H P and Nerurkar A B. Seroprevalence of Syphilis in Patient Attending Tertiary Care Hospital, Valsad, India. Int.J.Curr.Microbiol.App. Sci (2017) 6(6): 2702-2706

6. World Health Organization, Department of HIV/AIDS. 2001. Global prevalence and incidence of selected curable sexually transmitted diseases: overview and estimates. Geneva: World Health Organization.

7. Faiz NR, Malik L, Raziq F et al., Frequency of vaginal discharge and its association with various sexually transmitted diseases in women attending antenatal clinic. J Postgrad Med Institute 2005; 19: 86-95.

8. Newman L, Rowley J, Vander Hoorn S, Wijesooriya N S, Unemo M, Low N. 2015. Global Estimates of the Prevalence and Incidence of Four Curable Sexually Transmitted Infections in 2012 Based on Systematic Review and Global Reporting. PLoS ONE, 10(12): e0143304. doi:10.1371/journal.pone.014 3304.

9. Archana, B.R., Prasad, S.R., Beena, P.M., Okade, R., Sheela, S.R., Beeregowda, Y.C. 2014. Maternal and congenital syphilis in Karnataka, India. Southeast Asian J. Trop. Med. Public Health, 45(2): 430-43.

10. Maity, S., Bhunia, S.C., Biswas, S., Saha, M.K. 2011. Syphilis Seroprevalence among patients attending a sexually transmitted disease clinic in West Bengal, India. Jpn. J. Infect. Dis., 64(6): 506-508.

11. Schneider, J.A., Lakshmi, V., Dandona, R., et al., (2010): Population-based seroprevalence of HSV-2 and syphilis in Andhra Pradesh state of India. BMC Infect. Dis., 10, 59.

12. Müller, I., Brade, V., Hagedorn, H.J., Straube, E., Schörner, C., Frosch, M. 2014. Is serological testing a reliable tool in laboratory diagnosis of syphilis? Meta-analysis of eight external quality control surveys performed by the German infection serology proficiency testing program. J. Clin. Microbiol., 44: 1335-41.

13. Centers for Disease Control and Prevention. 2010. Sexually Transmitted Diseases Treatment Guidelines, 2010. MMWR Morb. Mortal Wkly. Rep., 59: 27-8.

14. Winn W, Allen S, Janda W, Koneman E, Schreckenberger P, Procop G and Woods G (2005), "Spirochetal Infections", Koneman's Textbook of Diagnostic Microbiology, 6th Edition, Lippincott Willium \& Wilkins, pp. 1126-1133.

15. World Health Organization An overview of selected curable STDs. Syphilis estimates, 2001. WHO office of HIV/AIDS and STDs. Geneva: WHO, 2001.

16. Khan, S., G.A. Menezes, R. Dhodapkar, B.N. Harish. Seroprevalence of syphilis in patients attending a tertiary care hospital in Southern India. Asian Pac. J. Trop. Biomed.,2014, 4(12): 995-997.

17. Bala K, Chaudhary U, Arora A and Mittal S. Recent trends of seroprevalence of syphilis in apparently healthy population in a tertiary care centre. Int. J. Pharm. Med. \& Bio. Sc. 2012Vol. 1, No. 2, October 2012.66-73.

\section{How to cite this article:}

Parul Punia, Deepinder Singh, Kiran Bala, Aparna, Uma Chaudhary and Akshit Griwan. 2017. Recent Trends of Sero-Prevalence of Syphilis in Patients Attending a Tertiary Care Centre in North India. Int.J.Curr.Microbiol.App.Sci. 6(11): 882-886. doi: https://doi.org/10.20546/ijcmas.2017.611.102 